\title{
Pauli Paramagnetism in Metals with High Densities of States $^{* 1}$
}

\begin{abstract}
S. Foner
Francis Bitter National Magnet Laboratory, Massachusetts Institute of Technology, Cambridge, Massachusetts 02139
\end{abstract}

(October 10, 1969)

Because the Pauli paramagnetic susceptibility of a free electron gas, $\chi_{p}^{0}$ is proportional to the density of states $N(0)$ at the Fermi energy, it is expected that measurements of $\chi_{p}^{0}$ of metals and alloys can yield a reasonable measure of $N(0)$. If $\chi_{p}^{0}$ can be measured directly, then $N(0)$ can be determined with high accuracy. The major obstacle to this procedure is that the measured static susceptibility $\chi_{p}=\chi_{p}^{0} /(1-N(0) V)=\chi_{p}^{0} D$ (for the Stoner model) where $V$ is a measure of the electron-electron interaction potential. Unfortunately, $V$ is not easily determined experimentally, or theoretically, so that measurements of $\chi_{p}$ do not yield accurate measures of $N(0)$. More detailed (realistic) models involve additional parameters which are also not accurately determined. Thus direct measurements of $\chi_{p}$ can yield a measure of $N(0)$, but these values of $N(0)$ are subject to the uncertainties of various parameters in the theories. In this talk we discuss the present status of experiments and theories of $\chi_{p}$ in metals and alloys with low $N(0)$ where $D$ is generally near unity, and high $N(0)$ where $D$ is expected to be large. Comparisons with independent low-temperature electronic specific heat measurements are discussed and attempts to derive $N(0)$ from both susceptibility and specific heat are reviewed.

Many recent investigations of metals and alloys with high densities of states have been of great interest because such systems start to approach ferromagnetic order. The properties of $\mathrm{Pd}$ have been investigated extensively and very detailed band calculations now exist. The large exchange enhancements in this metal and its

\footnotetext{
*An invited paper presented at the 3d Materials Research Symposium, Electronic Density of States, November 3-6, 1969, Gaithersburg, Md.

${ }^{1}$ Supported by the U.S. Air Force Office of Scientific Research.
}

alloys have permitted considerable progress to be made. The properties of these systems are reviewed and estimates of $N(0)$ and $V$ are examined. Here qualitative and quantitative comparisons of various experiments can be made. The possibilities of applying large magnetic fields and thereby independently measuring $N(0)$ and small changes of $N(0)$ near the Fermi energy by such studies are discussed. Recent theories which examine the changes of $\chi_{p}$ with alloying are also compared with experimental results.

A few useful references for relevant recent papers concerning the susceptibility of nearly ferromagnetic metals and alloys are tabulated in references 1 to 4 . Recent detailed band calculations in $\mathrm{Pd}$ have been reported in references 5 to 8 . Several papers at this conference also deal with high densities of states materials.

\section{References}

[1] Foner, S., Freeman, A. J., Blum, N., Frankel, R. B., McNiff, E. J., Jr., and Praddaude, H., Phys. Rev. 181, 863 (1969).

[2] Foner, S., and McNiff, E. J., Jr., Phys. Rev. Letters 19, 1438 (1967).

[3] Foner, S., and McNiff, E. J., Jr., Phys. Letters 29A, 28 (1969).

[4] Doclo, R., Foner, S., and Narath, A., J. Appl. Phys. 40, 1206 (1969).

[5] Mueller, F. M., Freeman, A. J., Dimmock, J. O., and Furdyna, A. (to be published).

[6] Hodges, L., Ehrenreich, H., and Lang, N. D., Phys. Rev. 152, 505 (1966).

[7] Anderson, O. K., and Mackintosh, A. R., Solid State Comm. 6, 285 (1968).

[8] Misetich, A. A., and Watson, R. E., J. Appl. Phys. 40, 1211 (1969). 